\title{
Heterogeneous prognosis among KIT mutation types in adult acute myeloid leukemia patients with $\mathrm{t}(8 ; 21)$
}

\author{
Ya-Zhen Qin', Hong-Hu Zhu (1)', Qian Jiang ${ }^{1}$, Lan-Ping Xu', Hao Jiang ${ }^{1}$, Yu Wang ${ }^{1}$, Xiao-Su Zhao ${ }^{1}$, Yan-Rong Liu', \\ Xiao-Hui Zhang', Kai-Yan Liu' ${ }^{1}$ and Xiao-Jun Huang $\mathbb{1}^{1,2}$
}

Acute myeloid leukemia (AML) with $t(8 ; 21)$ is a heterogeneous disease ${ }^{1}$. Therefore, additional prognostic factors are needed in order to make risk-adapted treatment approaches. KIT mutations are the most common mutations of $\mathrm{t}(8 ; 21)$ AML patients, and a spectrum of mutations has been detected to date ${ }^{2-6}$. Limited by sample size or the screening method, previous studies have usually analyzed all types of mutations as a whole or just analyzed the most prevalent D816 mutation ${ }^{2-7}$. Thus, whether each type of mutation has similar adverse impacts remains unclear to date. A reflection is that the existence of the KIT mutation brings $\mathrm{t}(8 ; 21)$ AML from low to intermediate risk regardless of mutation type in the National Comprehensive Cancer Network guidelines ${ }^{8}$, whereas European LeukemiaNet has provided no further recommendation for those with a KIT mutation? Recently, Yui et al. ${ }^{10}$ showed that the D816 mutation had a poorer prognosis than other mutations. Thus, it is urgent to perform large-scale studies under modern treatment modes to comprehensively evaluate the prognoses of the individual KIT mutation types.

A total of 275 consecutive adult patients with $t(8 ; 21)$ AML who were diagnosed and received treatment in our institute from June 2005 to December 2017 were, retrospectively, evaluated. Totally, 150 patients (54.5\%) were male. The median age of the patients at diagnosis was 36 (range: 16-69) years. As we previously reported, induction chemotherapy comprised 1-2 cycles of induction with the

\footnotetext{
Correspondence: X-J. Huang (huangxiaojun@bjmu.edu.cn)

${ }^{1}$ Peking University People's Hospital, Peking University Institute of Hematology, Beijing Key Laboratory of Hematopoietic Stem Cell Transplantation, Beijing 100044, China

${ }^{2}$ Peking-Tsinghua Center for Life Sciences, Beijing 100871, China

These authors contributed equally: Ya-Zhen Qin, Hong-Hu Zhu.
}

" $3+7$ " regimen or the homoharringtonine, aclarubicin, and cytarabine regimen (homoharringtonine $2 \mathrm{mg} / \mathrm{m}^{2}$ per day, cytarabine $100 \mathrm{mg} / \mathrm{m}^{2}$, and aclarubicin $20 \mathrm{mg} /$ day on days $1-7)^{11,12}$. Among the 263 patients achieving complete remission (CR), 142 received the intermediatedose cytarabine-based chemotherapy, 13 received chemotherapy followed by autologous-hematopoietic stem cell transplantation (auto-HSCT), 108 received chemotherapy followed by allogeneic-HSCT (allo-HSCT, human leukocyte antigen-identical sibling donor, $n=43$; matched unrelated donor, $n=7$; haploidentical related donor, $n=58$ ) as postremission therapy ${ }^{13}$. Dasatinib were used in some patients with KIT mutation if RUNX1-RUNX1T1 reduction is less than 3-log after cycle 2 consolidation since 2015. Nine and one patients who relapsed after chemotherapy and auto-HSCT received allo-HSCT as salvage therapy. The study was approved by the Ethics Committee of the Peking University People's Hospital. Informed consent was obtained from all subjects in accordance with the Declaration of Helsinki. The cutoff date for the follow-up was April 15, 2018. As we previously reported, the complementary DNA was used to amplify KIT exons 17 and 8 and sequencing ${ }^{4}$, and TaqMan-based real-time quantitative polymerase chain reaction technology was used to detect RUNX1-RUNX1T1 transcript levels ${ }^{11}$. The survival functions were estimated using the Kaplan-Meier method and were compared using the log-rank test. The parameters with $P<0.20$ by the univariate analysis were entered into a multivariate model using a Cox proportional hazards model to identify the most statistically significant parameters associated with relapse free survival (RFS) and overall survival (OS). The SPSS 16.0 software package (SPSS Inc., Chicago, IL) and GraphPad Prism 5 
Table 1 KIT mutation patterns

\begin{tabular}{|c|c|}
\hline Type of mutation & Number of patients (\%) \\
\hline Sole mutation & $103(90.4 \%)$ \\
\hline Exon 17 & $92(80.7 \%)$ \\
\hline R815_D816delinsK & $1(0.9 \%)$ \\
\hline R815_D816insT & $1(0.9 \%)$ \\
\hline R815_D816insIR & $1(0.9 \%)$ \\
\hline D816A & $1(0.9 \%)$ \\
\hline $\mathrm{D} 816 \mathrm{H}$ & $8(7.0 \%)$ \\
\hline D816V & 37 (32.5\%) \\
\hline D816Y & $10(8.8 \%)$ \\
\hline D820G & $3(2.6 \%)$ \\
\hline N822K & $28(24.6 \%)$ \\
\hline $\mathrm{N} 822 \mathrm{Y}$ & $1(0.9 \%)$ \\
\hline A829P & $1(0.9 \%)$ \\
\hline Exon 8 & $11(9.6 \%)$ \\
\hline T417_D419Delins| & $1(0.9 \%)$ \\
\hline T417_D419delinsY & $1(0.9 \%)$ \\
\hline T417_R420DelinsG & $1(0.9 \%)$ \\
\hline Y418delinsFFW & $1(0.9 \%)$ \\
\hline Y418_D419delinsP & $1(0.9 \%)$ \\
\hline Y418_R420delinsSW & $1(0.9 \%)$ \\
\hline Y418_L421delinsTRVY & $1(0.9 \%)$ \\
\hline D419del & $1(0.9 \%)$ \\
\hline D419_R420delinsK & $2(1.8 \%)$ \\
\hline D419_L421DelinsVEV & $1(0.9 \%)$ \\
\hline Compound mutations & $11(9.6 \%)$ \\
\hline $\mathrm{D} 816 \mathrm{~V}+\mathrm{D} 816 \mathrm{H}$ & $2(1.8 \%)$ \\
\hline D816V + D816Y & $3(2.6 \%)$ \\
\hline D816V + D820G & $1(0.9 \%)$ \\
\hline D816V + D419del & $1(0.9 \%)$ \\
\hline D816V + T417_L421delinsLPRF & $1(0.9 \%)$ \\
\hline D816Y + N822K & $1(0.9 \%)$ \\
\hline D820G + N822K & $1(0.9 \%)$ \\
\hline D820G + D419del & $1(0.9 \%)$ \\
\hline Total & $114(100 \%)$ \\
\hline
\end{tabular}

(GraphPad Software Inc., La Jolla, CA) were used for the data analysis.

The median follow-up time was 20 (2-93) months. The 3-year RFS and OS rates were 61.5\% (95\% confidence interval (CI), 53.9-68.2\%) and $73.2 \%$ (95\% CI, $67.3-80.4 \%)$, respectively. Overall, 114 patients $(41.5 \%)$ had KIT mutations, and a total of 22 types of mutations were detected (Table 1). In all, 103 and 11 patients, respectively, had sole and compound mutations (combination of 2 types), and 104 (37.8\%) and 14 (5.1\%) patients had a KIT mutation in exon 17 and exon 8 (sole or compound), respectively. The most prevalent mutation was exon 17 D816 (57.0\% of the patients with KIT mutations), followed by the exon $17 \mathrm{~N} 822$, exon 8 deletion-insertion and exon 17 D820 mutations (27.2, 12.3 and $4.4 \%$ ). The one-course and two-course CR rates were similar between the patients with KIT mutations and no mutation $(P=1.0$ and 0.45$)$. Patients with a $K I T$ mutation had significantly lower 3-year RFS and OS rates than those with no mutation (RFS: $P=0.0002$, 49.3\% [95\% CI: 37.0-60.5\%] vs. $69.7 \%$ [95\% CI 59.9-77.6\%]; OS: $P=$ 0.0055, 67.1\% [95\% CI: 55.0-76.6\%] vs. $77.6 \%$ [95\% CI: 68.3-84.5\%]). Patients with sole D816V, D816Y, and D816H mutation had similar 3-year RFS and OS rates $(P$ $=0.57$ and 0.087 ). Patients with a sole D816 mutation had significantly lower 3-year RFS and OS rates than those with no mutation (RFS, $P<0.0001,33.7 \%$ [95\% CI: 17.3-50.9\%] vs. 69.7\% [95\% CI: 59.9-77.6\%], Fig. 1a; OS, $P<0.0001,54.9 \%$ [95\% CI: 37.9-69.1\%] vs. 77.6\% [95\% CI: 68.3-84.5\%], Fig. 1b); Similar results existed if the patients who underwent allo-HSCT were censored at the time of transplantation (RFS: $P<0.0001,19.4 \% \quad[95 \% \quad \mathrm{CI}$ : 1.6-52.3\%] vs. $57.7 \%$ [95\% CI: 43.8-69.3\%], Fig. 1c; OS: $P$ $=0.0003,53.7 \%$ [95\% CI: $23.9-76.3 \%]$ vs. $77.0 \%$ [95\% CI: 63.2-86.2\%], Fig. 1d). In addition, the 3-year RFS and OS rates were similar among the patients with the sole N822 mutation, the exon 8 mutation and no mutation (RFS: $P$ $=0.47,69.6 \%$ [95\% CI: 46.1-84.4\%] vs. $88.9 \%$ [95\% CI: 43.3-98.4\%] vs. 69.7\% [95\% CI: 59.9-77.6\%], Fig. 1a; OS: $P=0.70,71.9 \%$ [95\% CI: 42.7-88.0\%] vs. $83.3 \%$ [95\% CI: 27.3-97.4\%] vs. $77.6 \%$ [95\% CI: 68.3-84.5\%], Fig. 1b). Likewise, the 3-year RFS and OS rates were similar if censoring at the time of transplantation (RFS: $P=0.36$, $52.6 \%[95 \%$ CI: $18.5-78.3 \%]$ vs. $80.0 \%\left[\begin{array}{lll}95 \% & \mathrm{CI}:\end{array}\right.$ 20.4-96.9\%] vs. 57.7\% [95\% CI: 43.8-69.3\%], Fig. 1c; OS: $P=0.32$, 0 [95\% CI: 0-0] vs. $75.0 \%$ [95\% CI: 12.8-96.1\%] vs. $77.0 \%$ [95\% CI: $63.2-86.2 \%$ ], Fig. 1d). Because of the similar prognoses for the N822 and exon 8 mutations compared to no mutation, five patients with sole or compound D820 mutation (Table 1) were analyzed together. Patients with the D820 mutation had significantly lower 3-year RFS rates than those with no mutation despite of no censoring or censoring (no censoring: $P=0.0050,20.0 \%$ [95\% CI: $0.8-58.2 \%$ ] vs. $69.7 \%$ [95\% CI: 59.9-77.6\%], Fig. 1a; censoring: $P<0.0001,0 \%$ [95\% CI: 0-0\%] vs. 57.7\% [95\% CI: 43.8-69.3\%], Fig. 1c). However, the D820 mutation had no impact on OS $(P=$ 0.73 and 0.72 , Fig. $1 b$ and $d)$.

Next, patients with KIT D816 and D820 mutations were defined as the D816/D820 mutation group ( $n=70,25.5 \%)$, 

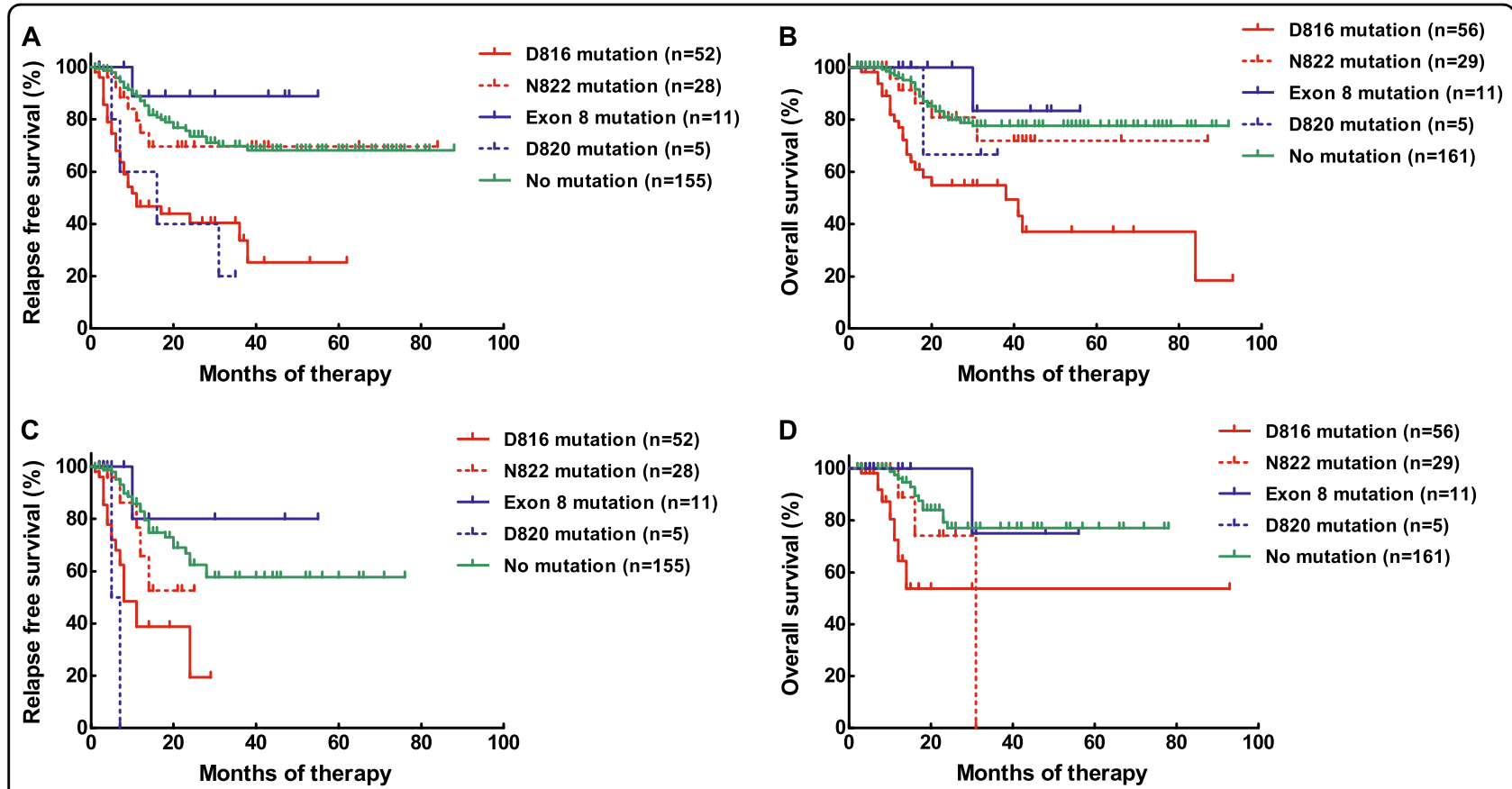

Fig. 1 RFS and OS of patients grouped by KIT mutation status and type. a RFS, no censoring. b OS, no censoring. $\mathbf{c}$ RFS, censoring at the time of allo-HSCT. d OS, censoring at the time of allo-HSCT

whereas the N822 and exon 8 mutation and no mutation were defined as the N822/exon 8/no mutation group $(n=$ 201, 73.1\%). Patients with the D816/D820 mutation had significantly lower 3-year RFS and OS rates than those with the N822/exon 8/no mutation (RFS: $P<0.0001,33.1 \%$ [95\% CI: $18.7-48.2 \%]$ vs. $70.5 \%$ [95\% CI: $61.9-77.5 \%]$; OS: $P<$ $0.0001,60.5 \%$ [95\% CI: $45.3-72.7 \%]$ vs. $77.2 \%$ [95\% CI: 68.8-83.6\%]). Similar results existed when censoring (RFS: $P<0.0001,0 \% \quad[95 \%$ CI: $0-0 \%]$ vs. $57.9 \% \quad[95 \%$ CI: 45.2-68.7\%]; OS: $P=0.0002,58.5 \%$ [95\% CI: 31.9-77.7\%] vs. $73.6 \%$ [95\% CI: $60.3-83.1 \%]$ ). Multivariate analyses showed that the KIT D816/D820 mutation, a <3-log reduction in the RUNX1-RUNX1T1 transcript levels at cycle 2 consolidation and treatment with chemotherapy only/auto-HSCT were independent adverse prognostic factors for both RFS and OS (Table S1).

In accordance with the majority of previous studies ${ }^{2-7}$, we confirmed that both the KIT mutation and the KIT D816 mutation were significantly associated with lower RFS and OS rates in adult $t(8 ; 21)$ AML. We also showed that the three common D816 mutations had similar clinical impacts. Furthermore, we demonstrated that the N822 and exon 8 mutations had similar RFS and OS rates compared to no mutation, whereas the D820 mutation had a significantly higher relapse probability than no mutation. The results implied that we should stratify the patients not only according to the existence of the KIT mutation but also according to the type of mutation. After regrouping, the KIT D816/D820 mutation was shown to be an independent adverse prognostic factor for both RFS and OS. The multivariate analyses result reflected that the pretreatment factor, treatment response and treatment modality were all relevant to the outcome of $t(8 ; 21)$ AML.

Consistent with the current clinical results, animal and in vitro studies show a functional difference between KIT mutations. Nick et al. ${ }^{14}$ used a murine model to illustrate that $K I T^{\mathrm{D} 814 \mathrm{~V}}$ promoted a more varied and aggressive leukemic phenotype than $K I T^{T 417 I \Delta 418-419}$ when coexpressed with RUNX1-RUNX1T1. Omori et al. demonstrated that in addition to the common JAK/STAT signaling pathway, the D816V mutation activated SRC family kinases, whereas N822K activated the MAPK pathway. The consequence was that D816V had a greater cell-proliferative and antiapoptotic ability than the N822K mutation $^{15}$.

The limitation was that this was a retrospective study. The treatment regimens were not uniform. Furthermore, we could not analyze the synergistic impact of the individual KIT mutations with other gene mutations due to lack of data.

In conclusion, the individual KIT mutations had distinct prognoses in adult $\mathrm{t}(8 ; 21)$ AML. Exon 17 D816 and D820 mutation had an adverse prognosis, whereas the exon 17 N822 and exon 8 mutation had a similar prognosis to no mutation. This result is helpful for a more precise stratification and for directing the appropriate treatment in $\mathrm{t}$ (8;21) AML. Multicenter prospective studies with a large sample size are warranted. 


\section{Acknowledgments}

This work was supported by the Natural Science Foundation of China (81570130 and 81570128) and the Foundation for Innovative Research Groups of the National Natural Science Foundation of China (81621001).

\section{Conflict of interest}

The authors declare that they have no conflict of interest.

\section{Publisher's note}

Springer Nature remains neutral with regard to jurisdictional claims in published maps and institutional affiliations.

Supplementary Information accompanies this paper at (https://doi.org/ 10.1038/s41408-018-0116-1).

Received: 21 May 2018 Revised: 1 July 2018 Accepted: 6 July 2018 Published online: 07 August 2018

\section{References}

1. Marcucci, G. et al. Prognostic factors and outcome of core-binding factor acute myeloid leukemia patients with $\mathrm{t}(8 ; 21)$ differ from those of patients with inv(16): a Cancer and Leukemia Group B study. J. Clin. Oncol. 23, 5705-5717 (2005).

2. Paschka, P. et al. Adverse prognostic significance of KIT mutations in adult acute myeloid leukemia with inv(16) and t(8;21): a Cancer and Leukemia Group B Study. J. Clin. Oncol. 24, 3904-3911 (2006).

3. Duployez, N. et al. Comprehensive mutational profiling of core-binding factor acute myeloid leukemia. Blood 127, 2451-2459 (2016).

4. Qin, Y. Z. et al. Prevalence and prognostic significance of c-KIT mutations in core-binding factor acute myeloid leukemia: a comprehensive large-scale study from a single Chinese center. Leuk. Res. 38, 1435-1440 (2014).
5. Cher, C. Y. et al. Next-generation sequencing with a myeloid gene panel in core-binding factor AML showed KIT activation loop and TET2 mutations predictive of outcome. Blood Cancer J. 6, e442 (2016).

6. Jang, W. et al. Significance of KIT exon 17 mutation depends on mutant level rather than positivity in core-binding factor acute myeloid leukemia. Blood Cancer J. 6, e387 (2016).

7. Schnittger, S. et al. KIT D816 mutations in AML1-ETO-positive AML are associated with impaired event-free and overall survival. Blood 107, 1791-1799 (2006).

8. National Comprehensive Cancer Network clinical practice guidelines in oncology (NCCN Guidelines): acute myeloid leukemia. Version 1. 2018.

9. Döhner, $H$. et al. Diagnosis and management of AML in adults: 2017 ELN recommendations from an international expert panel. Blood 129, 424-447 (2017).

10. Yui, S. et al. D816 mutation of the KIT gene in core-binding factor acute myeloid leukemia is associated with poorer prognosis than other KIT gene mutations. Ann. Hematol. 96, 1641-1652 (2017).

11. Zhu, H. H. et al. MRD-directed risk stratification treatment may improve outcomes of $\mathrm{t}(8 ; 21) \mathrm{AML}$ in the first complete remission: results from the AML05 multicenter trial. Blood 121, 4056-4062 (2013).

12. $\mathrm{Zhu}, \mathrm{H}$. H. et al. Homoharringtonine, aclarubicin and cytarabine (HAA) regimen as the first course of induction therapy is highly effective for acute myeloid leukemia with t (8;21). Leuk. Res. 44, 40-44 (2016).

13. Qin, Y. Z. et al. Low WT1 transcript levels at diagnosis predicted poor outcomes of acute myeloid leukemia patients with $\mathrm{t}(8 ; 21)$ who received chemotherapy or allogeneic hematopoietic stem cell transplantation. Chin. J. Cancer 35, 46 (2016).

14. Nick, H. J. et al. Distinct classes of c-Kit-activating mutations differ in their ability to promote RUNX1-ETO-associated acute myeloid leukemia. Blood $\mathbf{1 1 9}$ 1522-1531 (2012).

15. Omori, I. et al. D816V mutation in the KIT gene activation loop has greater cellproliferative and anti-apoptotic ability than N822K mutation in core-binding factor acute myeloid leukemia. Exp. Hematol. 52, 56-64.e4 (2017). 